\title{
On Constructions and Nonlinearity of Correlation Immune Functions (Extended Abstract)
}

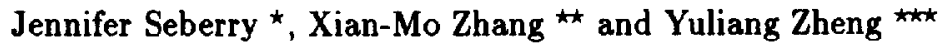 \\ Department of Computer Science, The University of Wollongong \\ Wollongong, NSW 2522, AUSTRALIA \\ E-mail: \{jennie, xianmo, yuliang\}ecs.uor.edu.au
}

\begin{abstract}
A Boolean function is said to be correlation immune if its output leaks no information about its input values. Such functions have many applications in computer security practices including the construction of key stream generators from a set of shift registers. Finding methods for easy construction of correlation immune functions has been an active research area since the introduction of the notion by Siegenthaler. In this paper we study balanced correlation immune functions using the theory of Hadamard matrices. First we present a simple method for directly constructing balanced correlation immune functions of any order. Then we prove that our method generates exactly the same set of functions as that obtained using a method by Camion, Carlet, Charpin and Sendrier. Advantages of our method over Camion et al's include (1) it allows us to calculate the nonlinearity, which is a crucial criterion for cryptographically strong functions, of the functions obtained, and (2) it enables us to discuss the propagation characteristics of the functions. Two examples are given to illustrate our construction method. Finally, we investigate methods for obtaining new correlation immune functions from known correlation immune functions. These methods provide us with a new avenue towards understanding correlation immune functions.
\end{abstract}

\section{Introduction}

The main component of a stream cipher is a key stream generator which produces from a random seed a sequence of pseudo-random bits. These pseudo-random bits are added modulo 2 to bits in a plaintext and the resulting stream, a ciphertext, is sent to a receiver. The receiver can recover the plaintext by adding modulo 2 to the ciphertext the output of the stream generator with the same seed.

* Supported in part by the Australian Research Council under the reference numbers A49130102, A9030136, A49131885 and A49232172.

* Supported in part by the Australian Research Council 16 under the reference number A49130102.

* Supported in part by the Australian Research Council under the reference number A49232172. 
A common method for obtaining key stream generators is to combine a set of shift registers with a nonlinear function. Blaser and Heinzmann [1] observed that if the combining function leaks information about its component functions, then the work needed in attacking the cryptosystem can be significantly reduced. This idea was further developed by Siegenthaler in [8] where a new concept called correlation immune functions was introduced. Since then the topic has been an active research area and correlation immunity has become one of the central design criteria for stream ciphers based on shift registers $[4,5]$.

For practical applications, finding methods for easy construction of correlation immune functions is of most importance. In [8] Siegenthaler presented the first method for constructing (balanced) correlation immune functions. His method is recursive in nature and hence not very satisfactory in practical applications. Camion et al studied correlation inmune functions from the point view of algebraic coding theory, and presented a method for constructing correlation immune functions of any order [2].

In this paper we study correlation immune functions using the theory of Hadamard matrices. First we present a method for directly constructing balanced correlation immune functions of any order. We then prove that our method generates exactly the same set of correlation immune functions as that obtained using Camion et al's method. Advantages of our method over Camion et al's include that, in addition to their orders of correlation immunity and algebraic degrees, it gives the nonlinearity and propagation characteristics of the functions obtained. We also study methods for constructing correlation immune functions on a higher dimensional space by combining known correlation immune functions on a lower dimensional space. The nonlinearity of functions thus constructed is also investigated.

The organization of the rest of the paper is as follows. Section 2 introduces notations and definitions that are needed in the paper. Section 3 reviews the previous construction methods for correlation immune functions. Our new construction method is described in Section 4. In the same section we also prove that the new construction method generates exactly the same set of correlation immune functions as that by Camion et al's method. Section 5 discusses the algebraic degree, nonlinearity and propagation characteristics of functions obtained using the new method. Two examples are shown in the same section. Section 6 is devoted to the combination of known correlation immune functions. Three combination methods are shown in the section, among which the first one can be viewed as an extension of the new construction method described in Section 4. The paper concludes with some remarks in Section 7.

\section{Preliminaries}

We consider $V_{m}$, the vector space of $m$ tuples of elements from $G F(2)$. Note that there is a natural one to one correspondence between vectors in $V_{m}$ and integers in $\left[0,2^{m}-1\right]$. This allows us to order the vectors according to their corresponding 
integer values. For convenience, we denote by $\alpha_{i}$ the vector in $V_{m}$ whose integer representation is $i$.

Let $f$ be a function from $V_{m}$ to $G F(2)$ (or simply a function on $V_{m}$ ). Since $f$ can be expressed as a unique polynomial in $m$ coordinates $x_{1}, x_{2}, \ldots, x_{m}$, we will identify $f$ with its unique multi-variable polynomial $f(x)$ where $x=$ $\left(x_{1}, x_{2}, \ldots, x_{m}\right)$. To distinguish between a vector of coordinates and an individual coordinate, the former will be strictly denoted by $w, x, y$ or $z$, while the later strictly by $w_{i}, x_{i}, y_{i}, z_{i}$ or $u$, where $i$ is an index. The algebraic degree of $f$ is defined as the number of coordinates in its longest term when it is represented in the algebraic normal form. $f$ is called an affine function if it takes the form of $f(x)=a_{1} x_{1} \oplus \cdots \oplus a_{m} x_{m} \oplus c$, where $a_{j}, c \in G F(2)$. In particular, $f$ is called a linear function if $c=0$.

The sequence of $f$ on $V_{m}$ is a $(1,-1)$-sequence defined by $\left((-1)^{f\left(\alpha_{0}\right)},(-1)^{f\left(\alpha_{1}\right)}\right.$, $\left.\ldots,(-1)^{f\left(\alpha_{2 m}-1\right)}\right)$, and the truth table of $f$ is a $(0,1)$-sequence defined by $\left(f\left(\alpha_{0}\right)\right.$, $\left.f\left(\alpha_{1}\right), \ldots, f\left(\alpha_{2 m}-1\right)\right) . f$ is said to be balanced if the truth table of $f$ has $2^{m-1}$ zeros (ones).

The following notation will be used in this paper. Let $\alpha=\left(a_{1}, \cdots, a_{m}\right)$ and $\beta=\left(b_{1}, \cdots, b_{m}\right)$ be two vectors (or sequences), the scalar product of $\alpha$ and $\beta$, denoted by $\langle\alpha, \beta\rangle$, is defined as the sum of the component-wise multiplications. In particular, when $\alpha$ and $\beta$ are from $V_{m},\langle\alpha, \beta\rangle=a_{1} b_{1} \oplus \cdots \oplus a_{m} b_{m}$, where the addition and multiplication are over $G F(2)$, and when $\alpha$ and $\beta$ are $(1,-1)$ sequences, $\langle\alpha, \beta\rangle=\sum_{i=1}^{m} a_{i} b_{i}$, where the addition and multiplication are over the reals.

Now we introduce the concept of correlation immune functions, the central topic treated in this paper. Let $f$ be a function on $V_{m}$. Let $X$ be a random variable taking on values $x \in V_{m}$ with uniform probability $2^{-m}$, let $X_{i}$ be the random variable corresponding to the $i$ th coordinate value $x_{i} \in G F(2)$, and let $Y$ be the random variable produced by the function $f$, i.e., $Y=f(X)$. $f$ is said to be a kth-order correlation immune function if the random variable $Y$ is statistically independent of any subset $X_{i_{1}}, X_{i_{2}}, \ldots, X_{i_{k}}$ of $k$ coordinates [8].

$\mathrm{Xiao}$ and Massey gave an equivalent definition for correlation immunity in terms of Walsh transformations [3]. The Walsh transformation $\hat{f}$ of a function $f$ on $V_{m}$ is defined as the real-valued function

$$
\hat{f}(\beta)=\sum_{x \in V_{m}} f(x)(-1)^{(\beta, x)},
$$

where $\beta \in V_{m}$. Note that in the sum, $f(x)$ and $\langle\beta, x\rangle$ are regarded as real-valued functions.

Definition 1. Let $f$ be a function on $V_{m} . f$ is a $k$ th-order correlation immune function if its Walsh transformation satisfies $\hat{f}(\beta)=0$ for all $\beta \in V_{m}$ with $1 \leq W(\beta) \leq k$, where $W(\beta)$ indicates the Hamming weight of, i.e., the number of the nonzero components in, a vector $\beta$.

A relevant topic, correlation immune functions with memory, was studied in [4]. The next lemma is useful for constructing correlation immune functions with a view to using Hadamard matrices. 
Lemma 2. Let $g$ be a function on $V_{m}$ and let $\eta$ be its sequence. Also let $x=$ $\left(x_{1}, x_{2}, \ldots, x_{m}\right)$. Then $g$ is a $k$ th-order correlation immune function if and only if $\langle\eta, \ell\rangle=0$ for any $\ell$, where $\ell$ is the sequence of a linear function $h(x)=\langle\alpha, x\rangle$ on $V_{m}$ constrained by $1 \leq W(\alpha) \leq k$.

Proof. Note that

$$
\begin{aligned}
\langle\eta, \ell\rangle & =\sum_{x \in V_{m}}(-1)^{g(x)}(-1)^{h(x)}=\sum_{x \in V_{m}}(-1)^{g(x)+(\alpha, x)} \\
& =\sum_{x \in V_{m}}(-1)^{\langle\alpha, x\rangle}-2 \sum_{x \in V_{m}} g(x)(-1)^{\langle\alpha, x\rangle} \\
& =-2 \hat{g}(\alpha) .
\end{aligned}
$$

Thus $\langle\eta, \ell\rangle=0$ if and only if $\hat{g}(\alpha)=0$ (See also Section 4.2, [2]).

The order $k$ of correlation immunity of a function on $V_{m}$ and its algebraic degree $d$ are constrained by the relation $k+d \leq m$. The only functions on $V_{m}$ that achieve the maximum $(m-1)$ th-order correlation immunity are $g\left(x_{1}, \ldots, x_{m}\right)=$ $x_{1} \oplus \cdots \oplus x_{m}$ and $g\left(x_{1}, \ldots, x_{m}\right)=x_{1} \oplus \cdots \oplus x_{m} \oplus 1$, both of which are affine. For balanced functions, if $k \neq 0$ or $m-1$, the relation becomes $k+d \leq m-1$ [8].

Next we introduce a fundamental combinatorial structure, the Hadamard matrix. Properties of Hadamard matrices will be very useful in our constructions of correlation immune functions. A $(1,-1)$-matrix $H$ of order $m$ is called a Hadamard matrix if $H H^{T}=m I_{m}$, where $H^{T}$ indicates the transpose of $H$ and $I_{m}$ is the identity matrix of order $m$. It is well known that the order $m$ of an Hadamard matrix is 1,2 or divisible by $4[9,6]$. In this paper we will use a special kind of Hadamard matrices called Sylvester-Hadamard matrices or Walsh-Hadamard matrices. A Sylvester-Hadamard matrix (or Walsh-Hadamard matrix) of order $2^{m}$, denoted by $H_{m}$, is generated by the following recursive relation

$$
H_{0}=1, H_{m}=\left[\begin{array}{rr}
1 & 1 \\
1 & -1
\end{array}\right] \otimes H_{m-1}, m=1,2, \ldots
$$

where denotes the Kronecker product. Note that $H_{m}$ can be written as $H_{m}=$ $H_{\imath} \diamond H_{t}$ for any nonnegative integers $s$ and $t$ with $s+t=m$. Sylvester-Hadamard matrices are closely related to linear functions, as is shown in the following lemma.

Lemma 3. Write $H_{m}=\left[\begin{array}{l}\ell_{0} \\ \ell_{1} \\ \vdots \\ \ell_{2^{m}-1}\end{array}\right]$ where $\ell_{i}$ is a row of $H_{m}$. Then $\ell_{i}$ is the sequence of a linear function $h_{i}=\left\langle\alpha_{i}, x\right\rangle$, where $x=\left(x_{1}, \ldots, x_{m}\right)$ and $\alpha_{i}$ is a vector in $V_{m}$ as defined in the first paragraph of this Section. Conversely the sequence of any linear function on $V_{m}$ is a row of $H_{m}$. 
A proof for the first half of the lemma can be found in [7]. The second half is true by noting the fact that $H_{m}$ has $2^{m}$ distinct rows and that there are exactly $2^{m}$ distinct linear functions on $V_{m}$. Thus the rows of $\pm H_{m}$ comprise all the affine sequences of length $2^{m}$.

Next we introduce a notation which is used throughout the rest of the paper. Given any vector $\delta=\left(i_{1}, \ldots, i_{s}\right) \in V_{s}$, we define a function on $V_{s}$ by

$$
D_{\delta}(y)=\left(y_{1} \oplus \overline{i_{1}}\right) \cdots\left(y_{s} \oplus \overline{i_{s}}\right)
$$

where $y=\left(y_{1}, \ldots, y_{0}\right)$ and $\vec{i}=1 \oplus i$ indicates the binary complement of $i$. Note that since $D_{\delta}(y)=1$ if and only if $y=\delta$, a function $f$ on $V_{s+t}$ can be expressed as

$$
f(y, x)=\bigoplus_{\delta \in V_{.}} D_{\delta}(y) f(\delta, x)
$$

where $x=\left(x_{1}, \ldots, x_{t}\right)$.

Lemma 4. Let $f(y, x)=\bigoplus_{\delta \in V} D_{\delta}(y) f_{\delta}(x)$ and $g(y, x)=\bigoplus_{\delta \in V_{0}} D_{\delta}(y) g_{\delta}(x)$ where $y=\left(y_{1}, \ldots, y_{s}\right)$, and $x=\left(x_{1}, \ldots, x_{t}\right)$. Then $f=g$ if and only if $f_{\delta}=g_{\delta}$ for all $\delta \in V_{8}$.

Proof. $f=g$ if and only if $f(\delta, x)=g(\delta, x)$ for all $\delta \in V_{s}$. Note that since $D_{\delta}(y)=1$ if and only if $y=\delta$, we have $f(\delta, x)=f_{\delta}(x)$ and $g(\delta, x)=g_{\delta}(x)$ for all $\delta \in V_{s}$.

The following lemma can be found in [7].

Lemma 5. Let $\xi_{i_{1} \ldots i_{p}},\left(i_{1}, \ldots, i_{p}\right) \in V_{p}$, be the sequence of a function $f_{i_{1} \ldots i_{p}}\left(x_{1}, \ldots, x_{q}\right)$ on $V_{q}$. Let $\xi$ be the concatenation of $\xi_{0} \ldots 00, \xi_{0} \ldots 01, \ldots, \xi_{1} \ldots 11$, namely, $\xi=$ $\left(\xi_{0} \ldots 00, \xi_{0} \ldots 01, \ldots, \xi_{1 \cdots 11}\right)$. Then $\xi$ is the sequence of a function on $V_{q+p}$ given by

$$
f\left(y_{1}, \ldots, y_{p}, x_{1}, \ldots, x_{q}\right)=\bigoplus_{\left(i_{1} \ldots i_{p}\right) \in V_{p}} D_{i_{1} \cdots i_{p}}\left(y_{1}, \ldots, y_{p}\right) f_{i_{1} \ldots i_{p}}\left(x_{1}, \ldots, x_{q}\right) .
$$

Let $\alpha=\left(a_{1}, a_{2}, \ldots, a_{n}\right) \in V_{n}$ and $\beta=\left(b_{1}, b_{2}, \ldots, b_{m}\right) \in V_{m}$. The Kranecker product of $\alpha$ and $\beta$, denoted by $\alpha \otimes \beta$, is defined as $\alpha \otimes \beta=\left(a_{1} \beta, a_{2} \beta, \ldots, a_{m} \beta\right)$. The following lemma will be used in the rest of the paper.

Lemma 6. Let $\xi$ be the sequence (or truth table) of a function $f$ on $V_{n}$ and $\eta$ be the sequence (or truth table) of a function $g$ on $V_{m}$. Then $\xi \otimes \eta$ is the sequence (or truth table) of the function $\varphi(y, x)=f(y) \oplus g(x)$ on $V_{n+m}$.

Proof. For any fixed $y=\alpha \in V_{n}$, we have $\varphi(\alpha, x)=f(\alpha) \oplus g(x)$.

The propagation characteristic is another nonlinearity measure for cryptographic functions. A function satisfies the propagation criterion of order $k$ if complementing $\boldsymbol{k}$ or less input coordinates results in the output being complemented half the times over all input vectors. The formal definition for the propagation criterion follows. 
Definition 7. Let $f$ be a function on $V_{n}$. We say that $f$ satisfies

1. the propagation criterion with respect to a non-zero vector $\alpha$ in $V_{n}$ if $f(x) \oplus$ $f(x \oplus \alpha)$ is a balanced function.

2. the propagation criterion of degree $k$ if it satisfies the propagation criterion with respect to all $\alpha \in V_{n}$ with $1 \leq W(\alpha) \leq k$.

\section{Previous Constructions}

Siegenthaler presented a recursive construction in his pioneering work [8]. Let $f_{1}$ and $f_{2}$ be $k$ th-order correlation immune functions on $V_{m}$. Then the concatenation of their sequences results in a new correlation immune function, namely,

$$
f(u, x)=(u \oplus 1) f_{1}(x) \oplus u f_{2}(x)
$$

is a $k$ th-order correlation immune function on $V_{m+1}$, where $u$ is a variable on $G F(2)$ and $x=\left(x_{1}, x_{2}, \ldots, x_{m}\right)$.

Camion et al [2] observed that in Siegenthaler's construction, if the Walsh transformations of $f_{1}$ and $f_{2}$ satisfy the condition

$$
\hat{f}_{1}(\lambda)+\hat{f}_{2}(\lambda)=0, \text { for all } \lambda \in V_{m} \text { with } W(\lambda)=k,
$$

then the order of the correlation immunity of $f$ is improved to $k+1$. In particular, they show the following two pairs of functions satisfy the condition:

1. $g(x)$ and $1 \oplus g(x)$;

2. $g(x)$ and $g(\bar{x})$, where $\bar{x}=\left(1 \oplus x_{1}, 1 \oplus x_{2}, \ldots, 1 \oplus x_{m}\right)$;

where $g$ is a $k$ th-order correlation immune function on $V_{m}$. Note that $1 \oplus g(x)$ complements the output, while $g(\bar{x})$ complements the input. Therefore, both

$$
f(x)=(u \oplus 1) g(x) \oplus u(1 \oplus g(x))=u \oplus g(x)
$$

and

$$
f(x)=(u \oplus 1) g(x) \oplus u g(\bar{x})=g(x) \oplus u(g(x) \oplus g(\bar{x}))
$$

are $(k+1)$ th-order correlation immune functions on $V_{m+1}$.

In the same paper, Camion et al also discovered a method for direct construction of correlation immune functions. Let $m$ and $n$ be positive integers with $m>n$. Let $r$ and $p_{j}, j=1,2, \ldots, n$ be arbitrary functions on $V_{m-n}$. Also let $x=\left(x_{1}, x_{2}, \ldots, x_{n}\right)$ and $y=\left(y_{1}, y_{2}, \ldots, y_{m-n}\right)$. Set

$$
f(y, x)=\bigoplus_{j=1}^{n} x_{j} p_{j}(y) \oplus r(y) .
$$

Then the function $f$ defined in (4) is a balanced $k$ th-order correlation immune function on $V_{m}$, where $k$ is an integer satisfying $k \geq \min \left\{W(P(y)) \mid y \in V_{m-n}\right\}-$ 1 , and $P(y)=\left(p_{1}(y), p_{2}(y), \ldots, p_{n}(y)\right)$. 


\section{A New Construction}

Let $m$ and $n$ be positive integers with $m>n$. Suppose that $\Phi_{m, n}=\left\{\varphi_{0} \ldots 0, \varphi_{0} \cdots 1, \ldots, \varphi_{1} \ldots 1\right\}$ is a set containing $2^{m-n}$ linear functions on $V_{n}$, each is indexed by a vector in $V_{m-n} . \Phi_{m, n}$ can be a multi-set and hence a linear function is allowed to appear more than once in $\Phi_{m, n}$. Let $x=\left(x_{1}, x_{2}, \ldots, x_{n}\right), y=\left(y_{1}, y_{2}, \ldots, y_{m-n}\right)$ and $r$ be an arbitrary function on $V_{m-n}$. Set

$$
g(y, x)=\bigoplus_{\delta \in V_{m-n}} D_{\delta}(y) \varphi_{\delta}(x) \oplus r(y)
$$

The following corollary is a consequence of Theorem 9 and Corollary 10 to be stated below, though it can be proved directly.

Corollary 8. The function $g$ defined in (5) is a balanced $k$ th-order correlation immune function on $V_{m}$, where $k$ is an integer satisfying $k \geq \min \left\{W\left(\gamma_{\delta}\right) \mid \delta \in\right.$ $\left.V_{m-n}\right\}-1, \varphi_{\delta}(x)=\left\langle\gamma_{\delta}, x\right\rangle \in \Phi_{m, n}$ and $\gamma_{\delta} \in V_{n}$.

Theorem 9. The constructions (4) and (5) express the same set of functions.

Proof. Let $S_{1}$ be the set of functions generated by (4) and $S_{2}$ the set of functions generated by $(5)$.

First we prove that $S_{1} \subseteq S_{2}$ by showing that a function obtained by (4) can always be represented in the form of (5). Let

$$
f(y, x)=\bigoplus_{j=1}^{n} x_{j} p_{j}(y) \oplus r(y)
$$

be a function in $S_{1}$. For any $\delta \in V_{m-n}$ we have

$$
f(\delta, x)=\bigoplus_{j=1}^{n} x_{j} p_{j}(\delta) \oplus r(\delta)
$$

Since $p_{j}(\delta) \in G F(2), j=1, \ldots, n, \bigoplus_{j=1}^{n} x_{j} p_{j}(\delta)$ is a linear function on $V_{n}$. Now let

$$
\varphi_{\delta}(x)=\bigoplus_{j=1}^{n} x_{j} p_{j}(\delta)
$$

and let

$$
g(y, x)=\bigoplus_{\delta \in V_{m-n}} D_{\delta}(y) \varphi_{\delta}(x) \oplus r(y) .
$$

Note that $D_{\delta}(y)=1$ if and only if $y=\delta$. Thus we have

$$
g(\delta, x)=\varphi_{\delta}(x) \oplus r(\delta)=f(\delta, x) .
$$

Since $\delta$ is arbitrary, by Lemma 4 we have

$$
f(y, x)=g(y, x) .
$$


Consequently, $f(y, x)$ can be represented in the form of (5). This means that $S_{1} \subseteq S_{2}$.

Next we show that a function obtained by (5) can be represented in the form of (4). This will prove that $S_{2} \subseteq S_{1}$. Let

$$
g(y, x)=\bigoplus_{\delta \in V_{m-n}} D_{\delta}(y) \varphi_{\delta}(x) \oplus r(y)
$$

be a function in $S_{2}$. Let $\delta$ be an arbitrary vector in $V_{m-n}$, and let

$$
\varphi_{\delta}(x)=a_{\delta, 1} x_{1} \oplus \cdots \oplus a_{\delta, n} x_{n}
$$

Now let $p_{j}, j=1,2, \ldots, n$, be a function on $V_{m-n}$ such that

$$
p_{j}(\delta)=a_{\delta, j}
$$

for all $\delta \in V_{m-n}$. Also let $P=\left(p_{1}, \ldots, p_{n}\right)$ be a mapping from $V_{m-n}$ to $V_{n}$ such that

$$
P(\delta)=\left(p_{1}(\delta), \ldots, p_{n}(\delta)\right)
$$

for all $\delta \in V_{m-n}$. Now we define a function on $V_{m}$ in the following way

$$
f(y, x)=\bigoplus_{j=1}^{n} x_{j} p_{j}(y) \oplus r(y) .
$$

Again since $D_{\delta}(y)=1$ if and only if $y=\delta$, we have

$$
g(\delta, x)=\varphi_{\delta}(x) \oplus r(\delta) .
$$

By (6) and (7) we have

$$
f(\delta, x)=\bigoplus_{j=1}^{n} x_{j} p_{j}(\delta) \oplus r(\delta)=\bigoplus_{j=1}^{n} x_{j} a_{\delta, j} \oplus r(\delta)=\varphi_{\delta}(x) \oplus r(\delta)=g(\delta, x) .
$$

Since $\delta$ is arbitrary, by Lemma 4 we have

$$
g(y, x)=f(y, x) .
$$

This implies that $g(y, x)$ can be presented in the form of (4) and thus $S_{2} \subseteq S_{1}$. This completes the proof that $S_{1}=S_{2}$.

Corollary 10. In the proof of Theorem 9

$$
\min \left\{W(P(y)) \mid y \in V_{m-n}\right\}-1=\min \left\{W\left(\gamma_{\delta}\right) \mid \delta \in V_{m-n}\right\}-1 .
$$

where $\varphi_{\delta}(x)=\left\langle\gamma_{\delta}, x\right\rangle=a_{\delta, 1} x_{1} \oplus \cdots \oplus a_{\delta, n} x_{n}$ and $\gamma_{\delta}=\left(a_{\delta, 1}, \ldots, a_{\delta, n}\right)$ are the same as in the proof of Theorem 9.

Proof. From (7) we have $P(\delta)=\left(a_{\delta, 1}, \ldots, a_{\delta, n}\right)$, and from (6) we have $\varphi_{\delta}(x)=$ $a_{\delta, 1} x_{1} \oplus \cdots \oplus a_{\delta, n} x_{n},=\left\langle\gamma_{\delta}, x\right\rangle$. Thus we have $P(\delta)=\gamma_{\delta}$ and hence $\min \{W(P(y)) \mid y \in$ $\left.V_{m-n}\right\}-1=\min \left\{W\left(\gamma_{\delta}\right) \mid \delta \in V_{m-n}\right\}-1$. 


\section{Applying the New Construction}

For integers $k$ and $n$ with $0 \leq k<n$, let $\Omega_{k, n}$ denote the set of linear functions on $V_{n}$ that have $k+1$ or more non-zero coefficients, namely

$$
\Omega_{k, n}=\left\{\varphi \mid \varphi(x)=\langle\beta, x\rangle, \beta \in V_{n}, W(\beta) \geq k+1\right\}
$$

where $x=\left(x_{1}, \ldots, x_{n}\right)$. This set of functions will be used in our constructions of correlation immune functions.

\subsection{Balanced Functions with Given Immunity}

Given two integers $m$ and $k$ with $m \geq 3$ and $1 \leq k<m-1$, balanced $k$ th-order correlation immune functions on $V_{m}$ can be constructed in the following way.

1. Fix an integer $n$ such that $k<n<m$.

2. Create a set $\Phi_{m, n}$ by selecting linear functions strictly from $\Omega_{k, n}$. Note that the size of $\Phi_{m, n}$ is $2^{m-n}$, and repetition is permitted in the selection.

3. Construct a function by using the method (5).

By Corollary 8 , we have

Theorem 11. A function constructed according to the above three steps is a balanced $k$ th-order correlation immune function on $V_{m}$.

\subsection{Algebraic Degrees}

Let $k$ and $m$ be integers with $k \geq 1$ and $m \geq k+2$. As mentioned in Section 2, the algebraic degree of a balanced $k$ th-order immune correlation functions on $V_{m}$ is at most $m-k-1$. We are interested in constructing balanced $k$ th-order correlation immune functions having the maximum algebraic degree $m-k-1$.

In order to discuss their algebraic degrees, we construct functions in the following three steps.

1. Fix an integer $n$ such that $m>n \geq k+2$.

2. Choose a multi-set $\Phi_{m, n}=\left\{\varphi_{\delta}: V_{n} \rightarrow G F(2) \mid \delta \in V_{m-n}\right\}$ of linear functions in such a way that it satisfies the following three conditions:

(C1) If $\varphi \in \Phi_{m, n}$ then $\varphi \in \Omega_{k, n}$, where $\Omega_{k, n}$ is defined in (8),

(C2) $\Phi_{m, n}$ contains at least two distinct functions,

(C3) there is a variable $x_{j}$ that appears in an odd number of functions in $\Phi_{m, n}$. Note that the repetition of functions is counted by the number of appearance.

3. Employ the set $\Phi_{m, n}$ in the construction (5).

Since $\Phi_{m, n}$ is a multi-set, the condition (C1) can be satisfied. On the other hand, since $n \geq k+2$ and $\Omega_{k, n}$ contains more than two functions, the condition (C2) can also be readily satisfied. 
Once the conditions (C1) and (C2) are satisfied, we check $\Phi_{m, n}$ to see if it satisfies the condition (C3). If not, we modify $\Phi_{m, n}$ in the following way. Since $\Phi_{m, n}$ satisfies the condition (C2), there are two distinct functions $\varphi_{\delta_{1}}(x), \varphi_{\delta_{2}}(x) \in$ $\Phi_{m, n}$. Thus there exists some $x_{j}$ that appears in $\varphi_{\delta_{1}}(x)$ but not in $\varphi_{\delta_{2}}(x)$. Now we replace $\varphi_{\delta_{2}}(x)$ by $\varphi_{\delta_{1}}(x)$. In this way we can modify the function set $\Phi_{m, n}$ so that it satisfies the condition (C3). When the condition (C3) is satisfied, there is a term $y_{1} \cdots y_{m-n} x_{j}$ that appears an odd number of times in a function $g$ constructed according to the above three steps. This term survives in the final algebraic normal form representation of $g$. In other words, the algebraic degree of $g$ is $m-n+1$.

From Theorem 11 and the above discussions, we know that $g$ is a balanced $k$ th-order correlation immune function of algebraic degree $m-n+1$. Thus we have proved

Theorem 12. Let $k, n$ and $m$ be integers with $k \geq 1$ and $m>n \geq k+2$. Then a function constructed according to the above three steps is a balanced kth-order correlation immune function on $V_{m}$ of algebraic degree $m-n+1$. When $n$ is chosen as $n=k+2$, the function achieve the maximum algebraic degree $m-k-1$.

\subsection{Nonlinearity}

Given two functions $f$ and $g$ on $V_{m}$, the Hamming distance between $f$ and $g$ is defined as $d(f, g)=W(f(x) \oplus g(x))$. The nonlinearity of $g$ is defined as $N_{f}=\min _{i=0,1, \ldots, 2^{m+1}-1} d(f, \varphi)$ where $\varphi_{0}, \varphi_{1}, \ldots, \varphi_{2^{m+1}-1}$ comprise all the affine functions on $V_{m}$. It has been proved that $N_{f} \leq 2^{m-1}-2^{\frac{m}{2}-1}$ for any function $f$ on $V_{m}$ [7]. Nonlinearity is an crucial criterion for cryptographic functions and it measures the ability of a cryptographic system using the functions to resist being expressed as a set of linear equations. If the system could be expressed as linear equations, it would be easily breakable by various attacks.

Let $f_{1}$ and $f_{2}$ be functions on $V_{m}, \xi_{1}$ and $\xi_{2}$ be the sequences of $f_{1}$ and $f_{2}$ respectively. Then $\left\langle\xi_{f}, \xi_{g}\right\rangle=\sum_{f(x)=g(x)} 1-\sum_{f(x) \neq g(x)} 1=2^{m}-2 \sum_{f(x) \neq g(x)} 1$ $=2^{m}-2 d(f, g)$. This proves the following result which is very useful in the study of the nonlinearity of functions.

Lemma 13. Let $f$ and $g$ be functions on $V_{m}$ whose sequences are $\xi_{f}$ and $\xi_{g}$ respectively. Then the distance between $f$ and $g$ can be calculated by $d(f, g)=$ $2^{m-1}-\frac{1}{2}\left\langle\xi_{f}, \xi_{g}\right\rangle$.

Now we calculate the nonlinearity of correlation immune functions constructed by $(5)$.

Theorem 14. Let $m$ and $n$ be integers with $m>n>2$, and let $g$ be a function constructed by (5). Denote by $t_{\delta}$ the number of times a linear function $\varphi_{\delta}$ appears in $\Phi_{m, n}$, and let $t=\max \left\{t_{\delta} \mid \delta \in V_{m-n}\right\}$. Then the nonlinearity of $g$ satisfies $N_{g} \geq 2^{m-1}-t 2^{n-1}$. 
Proof. For convenience a vector $\delta \in V_{m-n}$ will be denoted by its corresponding integer between 0 and $2^{m-n}-1$. In this way, a linear function $\varphi_{\delta} \in \Phi_{m, n}$ indexed by $\delta$ is rewritten as $\varphi_{j}$ and $t_{\delta}$ is rewritten as $t_{j}$, where $t_{\delta}$ is the number of times $\varphi_{\delta}$ appears in $\Phi_{m, n}$ and $j$ is the integer representation of $\delta$. We first consider the case when $r(y)=0$ in the construction (5), namely

$$
g(y, x)=D_{0 \cdots 0}(y) \varphi_{0}(x) \oplus \cdots \oplus D_{1 \cdots 1}(y) \varphi_{2^{m-n}-1}(x)
$$

where $\varphi_{j} \in \Omega_{k, n}, y=\left(y_{1}, \ldots, y_{m-n}\right), x=\left(x_{1}, \ldots, x_{n}\right)$, and $D_{j_{1} \cdots j_{m-n}}$ is defined in Section 2.

Let $h$ be any affine function on $V_{m}$. By Lemma 3 , the sequence of $h$, denoted by $L$, is a row of $\pm H_{m}$. Since $H_{m}=H_{m-n} \otimes H_{n}, L$ can be expressed as $L=$ $\pm \ell^{\prime} \otimes \ell^{\prime \prime}$, the Kronecker product of $\ell^{\prime}$ and $\ell^{\prime \prime}$, where $\ell^{\prime}$ is a row of $H_{m-n}$ while $\ell^{\prime \prime}$ is a row of $H_{n}$. Write $\ell^{\prime}$ as $\ell^{\prime}=\left(c_{0}, c_{1}, \ldots, c_{2^{m-n}-1}\right)$. Then $L$ can be rewritten as $L=\left(c_{0} \ell^{\prime \prime}, c_{1} \ell^{\prime \prime}, \ldots, c_{2^{m-n}-1} \ell^{\prime \prime}\right)$. Note that by Lemma $3, \ell^{\prime \prime}$ is the sequence of a linear function. We denote the linear function by $\varphi^{\prime \prime}$.

Now let $\zeta_{j}$ be the sequence of $\varphi_{j}, j=0,1, \ldots, 2^{m-n}-1$. By Lemma 5 , $\eta=\left(\zeta_{0}, \zeta_{1}, \ldots, \zeta_{2^{m-n}-1}\right)$ is the sequence of $g$ defined in (9). On the other hand, since the rows of an Hadamard matrix are mutually orthogonal, we have the following result:

$$
\left\langle\zeta_{j}, \ell^{\prime \prime}\right\rangle= \begin{cases}2^{n}, & \text { if } \varphi_{j}=\varphi^{\prime \prime} \\ 0, & \text { otherwise. }\end{cases}
$$

Now we discuss $\langle\eta, L\rangle$ in the following two cases:

Case 1: there exists a $j$ such that $\varphi_{j}=\varphi^{\prime \prime}$; since $\varphi_{j}$ appears $t_{j}$ times in $\Phi_{m, n}$, the total number of times when $\varphi_{j}=\varphi^{\prime \prime}$ is also $t_{j}$. Thus $|\langle\eta, L\rangle| \leq t_{j} 2^{n}$.

Case 2: there exists no $j$ such that $\varphi_{j}=\varphi^{\prime \prime}$; in this case we have $|\langle\eta, L\rangle|=0$.

Summarizing Cases 1 and 2 , we have $|\langle\eta, L\rangle| \leq t 2^{n}$. By Lemma $13, d(g, h) \geq$ $2^{m-1}-t 2^{n-1}$. Since $h$ is arbitrary, we have $N_{g} \geq 2^{m-1}-t 2^{n-1}$.

Now consider the more general case when $r(y) \neq 0$ in the construction (5). Since $r$ is a function of $y$ but not $x$, the sequence of $g$ takes the form of $\eta=$ $\left(e_{0} \zeta_{0}, e_{1} \zeta_{1}, \ldots, e_{2^{m-n-1}} \zeta_{2^{m-n}-1}\right)$, where $e_{i}=(-1)^{r\left(\alpha_{i}\right)}$ and $\alpha_{i}$ is a vector in $V_{m-n}$ whose integer representation is $i$. By a similar discussion to the case when $r(y)=0$, we have $|\langle\eta, L\rangle| \leq t 2^{n}$ for any affine sequence $L$, and hence $N_{g} \geq$ $2^{m-1}-t 2^{n-1}$.

\subsection{Propagation Characteristics}

This section discusses the propagation characteristics of functions obtained by (5). For convenience, the construction method is repeated here:

$$
g(y, x)=\bigoplus_{\delta \in V_{m-n}} D_{\delta}(y) \varphi_{\delta}(x) \oplus r(y)
$$

In the following discussion, we assume that all linear functions $\varphi_{\delta}$ in the construction are distinct. 
It is easy to prove that

$$
D_{\delta}(y \oplus \beta)=D_{\delta \oplus \beta}(y)
$$

Let $z=(y, x)$. Also let $\beta \in V_{m-n}, \alpha \in V_{n}$ and $\gamma=(\beta, \alpha)$. Then

$$
\begin{aligned}
g(z \oplus \gamma) & =\bigoplus_{\delta \in V_{m-n}} D_{\delta}(y \oplus \beta) \varphi_{\delta}(x \oplus \alpha) \oplus r(y \oplus \beta) \\
& =\bigoplus_{\delta \in V_{m-n}}(y) D_{\delta \oplus \beta}(y) \varphi_{\delta}(x \oplus \alpha) \oplus r(y \oplus \beta) \\
& =\bigoplus_{\delta \oplus \beta \in V_{m-n}} D_{\delta \oplus \beta}(y) \varphi_{\delta}(x \oplus \alpha) \oplus r(y \oplus \beta)
\end{aligned}
$$

Set $\sigma=\delta \oplus \beta$, we have

$$
g(z \oplus \gamma)=\bigoplus_{\sigma \in V_{m-n}} D_{\sigma}(y) \varphi_{\sigma \oplus \beta}(x \oplus \alpha) \oplus r(y \oplus \beta)
$$

and hence

$$
g(z) \oplus g(z \oplus \gamma)=\bigoplus_{\sigma \in V_{m-n}} D_{\sigma}(y)\left(\varphi_{\sigma}(x) \oplus \varphi_{\sigma \oplus \beta}(x \oplus \alpha)\right) \oplus r(y) \oplus r(y \oplus \beta)
$$

Note that for any fixed $y=\sigma$

$$
\left.(g(z) \oplus g(z \oplus \gamma))\right|_{y=\delta}=\varphi_{\sigma}(x) \oplus \varphi_{\sigma \oplus \beta}(x \oplus \alpha) \oplus r(\sigma) \oplus r(\sigma \oplus \beta)
$$

Consider the case when $\beta \neq(0, \ldots, 0)$. By assumption $\varphi_{\sigma}(x)$ and $\varphi_{\sigma \oplus \beta}(x)$ are distinct linear functions. Hence $\left.\varphi_{\sigma}(x) \oplus \varphi_{\sigma \oplus \beta}(x \oplus \alpha)\right)=\varphi_{\sigma}(x) \oplus \varphi_{\sigma \oplus \beta}(x) \oplus$ $\varphi_{\delta \oplus \beta}(\alpha)$ is a non-constant affine function which is balanced. This shows that $g(z) \oplus g(z \oplus \gamma)$ is balanced for any $\gamma=(\beta, \alpha)$ with $\beta \neq(0, \ldots, 0)$. Thus we have proved

Theorem 15. In the construction (5), if all $\varphi_{6}$ are distinct linear functions on $V_{n}$, then $g$ satisfies the propagation criterion with respect to all $\gamma$ with $\gamma=(\beta, \alpha)$, $\beta \in V_{m-n}, \alpha \in V_{n}$ and $\beta \neq 0$.

Note that there are $2^{m-n}-1$ choices for $\beta \neq 0$ and $2^{n}$ choices for all $\alpha \in V_{n}$. Therefore the total number of vectors with respect to which the function $g$ satisfies the propagation criterion is at least $\left(2^{m-n}-1\right) 2^{n}=2^{m}-2^{n}$. 


\subsection{Examples}

Theorem 12 gives us a general method to construct balanced correlation immune functions having any given immunity. The construction method allows us to easily calculate the algebraic degree and the nonlinearity of the functions, which is very desirable in designing cryptographic systems. Two concrete examples follow.

Let $n=4$ and $k=2$. Then

$$
\begin{aligned}
\Omega_{2,4} & =\left\{\varphi \mid \varphi(x)=\langle\beta, x\rangle, \beta \in V_{4}, W(\beta) \geq 3\right\} \\
& =\left\{x_{1} \oplus x_{2} \oplus x_{3}, x_{1} \oplus x_{2} \oplus x_{4}, x_{1} \oplus x_{3} \oplus x_{4}, x_{2} \oplus x_{3} \oplus x_{4}, x_{1} \oplus x_{2} \oplus x_{3} \oplus x_{4}\right\} .
\end{aligned}
$$

where $x=\left(x_{1}, x_{2}, x_{3}, x_{4}\right)$.

Example 1. We construct a balanced 2nd-order immune function $f$ on $V_{7}$, which achieves the maximum algebraic degree of 4 . We also calculate the nonlinearity of the function.

Set

$$
\begin{aligned}
& \varphi_{1}(x)=x_{1} \oplus x_{2} \oplus x_{3}, \varphi_{5}(x)=\varphi_{1}(x) \\
& \varphi_{2}(x)=x_{1} \oplus x_{2} \oplus x_{4}, \varphi_{6}(x)=\varphi_{2}(x) \\
& \varphi_{3}(x)=x_{1} \oplus x_{3} \oplus x_{4}, \varphi_{7}(x)=\varphi_{3}(x) \\
& \varphi_{4}(x)=x_{2} \oplus x_{3} \oplus x_{4}, \varphi_{8}(x)=\varphi_{3}(x)
\end{aligned}
$$

and

$$
\Phi_{7,4}=\left\{\varphi_{1}, \varphi_{2}, \varphi_{3}, \varphi_{4}, \varphi_{5}, \varphi_{6}, \varphi_{7}, \varphi_{8}\right\} .
$$

$\Phi_{7,4}$ is a multi-set whose elements are all taken from $\Omega_{2,4}$. In addition, it contains four different functions, and $x_{1}$ appears in seven functions. Thus the three conditions (C1), (C2) and (C3) are all satisfied.

To complete the construction, let

$$
\begin{aligned}
f(y, x)= & D_{000}(y) \varphi_{1}(x) \oplus D_{001}(y) \varphi_{2}(x) \oplus D_{010}(y) \varphi_{3}(x) \oplus D_{011}(y) \varphi_{4}(x) \oplus \\
& D_{100}(y) \varphi_{5}(x) \oplus D_{101}(y) \varphi_{6}(x) \oplus D_{110}(y) \varphi_{7}(x) \oplus D_{111}(y) \varphi_{8}(x) \\
= & \left(1 \oplus y_{2} y_{3} \oplus y_{1} y_{2} y_{3}\right) x_{1} \oplus\left(1 \oplus y_{2} \oplus y_{2} y_{3} \oplus y_{1} y_{2} y_{3}\right) x_{2} \oplus \\
& \left(1 \oplus y_{3} \oplus y_{2} y_{3}\right) x_{3} \oplus\left(y_{2} \oplus y_{3} \oplus y_{2} y_{3}\right) x_{4}
\end{aligned}
$$

where $y=\left(y_{1}, y_{2}, y_{3}\right)$ and $x=\left(x_{1}, x_{2}, x_{3}, x_{4}\right)$.

By Theorem 12, $f$ is a balanced $2 n$ d-order correlation immune function on $V_{7}$ of algebraic degree 4 . To calculate the nonlinearity of the function, note that $\varphi_{3}=\varphi_{7}=\varphi_{8}$ and hence $t=\max \left\{t_{j} \mid j=1, \ldots, 8\right\}=3$. By Theorem 14, we have $N_{f} \geq 2^{7-1}-3 \cdot 2^{4-1}=40$. Note that the upper bound of the nonlinearity of balanced functions on $V_{7}$ is 56 (see Corollary 17 of [7].

Example 2. In this example, we construct a balanced 2nd-order immune function $g$ on $V_{6}$. Let

$$
\begin{aligned}
& \varphi_{1}(x)=x_{1} \oplus x_{2} \oplus x_{3}, \\
& \varphi_{2}(x)=x_{1} \oplus x_{2} \oplus x_{4}, \\
& \varphi_{3}(x)=x_{1} \oplus x_{3} \oplus x_{4}, \\
& \varphi_{4}(x)=x_{1} \oplus x_{2} \oplus x_{3} \oplus x_{4}
\end{aligned}
$$


and

$$
\Phi_{6,4}=\left\{\varphi_{1}, \varphi_{2}, \varphi_{3}, \varphi_{4}\right\} .
$$

Obviously $\Phi_{6,4}$ satisfies the three conditions (C1), (C2) and (C3).

Let

$$
\begin{aligned}
g(y, x)= & D_{00}(y) \varphi_{1}(x) \oplus D_{01}(y) \varphi_{2}(x) \oplus D_{10}(y) \varphi_{3}(x) \oplus D_{11}(y) \varphi_{4}(x) \\
= & x_{1} \oplus\left(1 \oplus y_{1} \oplus y_{1} y_{2}\right) x_{2} \oplus \\
& \left(1 \oplus y_{2} \oplus y_{1} y_{2}\right) x_{3} \oplus\left(y_{1} \oplus y_{2} \oplus y_{1} y_{2}\right) x_{4}
\end{aligned}
$$

where $y=\left(y_{1}, y_{2}\right)$ and $x=\left(x_{1}, x_{2}, x_{3}, x_{4}\right)$.

$g$ is a balanced 2nd-order correlation immune function on $V_{6}$. It satisfies the propagation criterion with respect to all $\alpha=\left(a_{1}, a_{2}, a_{3}, a_{4}, a_{5}, a_{6}\right) \in V_{6}$ with $a_{1} \neq 0$ or $a_{2} \neq 0$. The algebraic degree of $g$ is 3 and the nonlinearity of $g$ is $N_{g} \geq 2^{6-1}-2^{4-1}=24$. For comparison, note that the upper bound for the nonlinearity of balanced functions on $V_{6}$ is 26 (see [7]).

\section{Combination of Correlation Immune Functions}

The construction (5) described in Section 4 presents a method for directly constructing correlation immune functions of any order. In this section we discuss three methods for constructing correlation immune functions on a higher dimensional space from existing such functions on a lower dimensional space.

\subsection{An Extension of the New Construction}

The construction (5) can be extended. Let $m, n, k$ and $s$ be positive integers, where $m>n>k$, and let $w=(y, x, z), y=\left(y_{1}, \ldots, y_{m-n}\right), x=\left(x_{1}, \ldots, x_{n}\right)$ and $z=\left(z_{1}, \ldots, z_{0}\right)$. Also let $\Phi_{m, n}=\left\{\varphi_{0}, \ldots, \varphi_{2^{m-n}-1}\right\}$ be a set of linear functions on $V_{n}$, each of which is selected from $\Omega_{k, n}$. Repetition is permitted in selecting the linear functions. Set

$$
g_{1}(y, x)=D_{0} \cdots 0(y) \varphi_{0}(x) \oplus \cdots \oplus D_{1 \cdots 1}(y) \varphi_{2^{m-n}-1}(x) \oplus r_{1}(y)
$$

where $r_{1}$ is an arbitrary function on $V_{m-n}$. By Corollary 8, $g_{1}$ is a balanced $k$ th-order correlation immune functions on $V_{m}$.

Now let $\left\{f_{0}, \ldots, f_{2^{m-n}-1}\right\}$ be a set of $p$ th-order correlation immune functions on $V_{s}$. Functions in the set need not be mutually distinct. Set

$$
g_{2}(y, z)=D_{0 \cdots 0}(y) f_{0}(z) \oplus \cdots \oplus D_{1 \cdots 1}(y) f_{2^{m-n}-1}(z) \oplus r_{2}(y)
$$

where $r_{2}$ is an arbitrary function on $V_{m-n}$. We further set

$$
g(y, x, z)=g_{1}(y, x) \oplus g_{2}(y, z)
$$


Theorem 16. The function $g(y, x, z)=g_{1}(y, x) \oplus g_{2}(y, z)$ is a balanced $(k+p+$ 1) th-order correlation immune function on $V_{m+\varepsilon}$. The nonlinearity of $g$ satisfies

$$
N_{g} \geq 2^{m-1}-t \cdot 2^{n}\left(2^{s-1}-N\right)
$$

where $t=\max \left\{t_{j} \mid j=0,1, \ldots, 2^{m-n}-1\right\}, t_{j}$ denotes the number of times that $\varphi_{j}$ appears in $\Phi_{m, n}$, and $N=\min \left\{N_{f_{j}} \mid j=0,1, \ldots, 2^{m-n}-1\right\}$.

Proof. We first consider the case when $r(y)=r_{1}(y) \oplus r_{2}(y)=0$. Note that $g(y, x, z)=D_{0 \cdots 0}(y)\left(\varphi_{0}(x) \oplus f_{0}(z)\right) \oplus \cdots \oplus D_{1 \cdots 1}(y)\left(\varphi_{2^{m-n}-1}(x) \oplus f_{2^{m-n}-1}(z)\right)$.

Since each $\varphi_{j}$ is balanced, each $\varphi_{j}(x) \oplus f_{j}(z)$ is also balanced (see Lemma 20 of [7]). Hence $g(y, x, z)$ is balanced.

Now we show that $g$ is a $(k+p+1)$ th-order correlation immune function. Let $\zeta_{j}$ and $\xi_{j}$ be the sequences of $\varphi_{j}$ and $f_{j}$ respectively, $j=0,1, \ldots, 2^{m-n}-1$. By Lemma $6 \zeta_{j} \otimes \xi_{j}$ is the sequence of $\varphi_{j}(x) \oplus f_{j}(z)$, and $\eta=\left(\zeta_{0} \otimes \xi_{0}, \ldots, \zeta_{2^{m-n}-1} \otimes\right.$ $\left.\xi_{2^{m-n}-1}\right)$ is the sequence of $g(y, x, z)$ (see Lemma 5 ).

Let $h$ be a linear function on $V_{m+s}$. By Lemma 3 , the sequence of $h$, denoted by $L$, is a row of $H_{m+s}$. Since $H_{m+s}=H_{m-n} \otimes H_{n} \otimes H_{s}, L$ can be expressed as $L=\ell_{1} \otimes \ell_{2} \otimes \ell_{3}$, where $\ell_{1}$ is a row of $H_{m-n}, \ell_{2}$ is a row of $H_{n}$, and $\ell_{3}$ is a row of $H_{3}$. Write $\ell_{1}=\left(c_{0}, c_{1}, \ldots, c_{2^{m-n}-1}\right)$. Then $L$ can be rewritten as $L=\left(c_{0} \ell_{2} \otimes \ell_{3}, \ldots, c_{2^{m-n}-1} \ell_{2} \otimes \ell_{3}\right)$. Let $\eta$ be the sequence of $g$. Then

$$
\begin{aligned}
\langle\eta, L\rangle & =c_{0}\left\langle\zeta_{0} \otimes \xi_{0}, \ell_{2} \otimes \ell_{3}\right\rangle+\cdots+c_{2^{m-n}-1}\left\langle\zeta_{2^{m-n}-1} \otimes \xi_{2^{m-n}-1}, \ell_{2} \otimes \ell_{3}\right\rangle \\
& =c_{0}\left\langle\zeta_{0}, \ell_{2}\right\rangle\left\langle\xi_{0}, \ell_{3}\right\rangle+\cdots+c_{2^{m-n}-1}\left\langle\zeta_{2^{m-n}-1}, \ell_{2}\right\rangle\left\langle\xi_{2^{m-n}-1}, \ell_{3}\right\rangle .
\end{aligned}
$$

Write $h(w)=\langle\gamma, w\rangle=\langle\beta, y\rangle \oplus\langle\alpha, x\rangle \oplus\langle\sigma, z\rangle$, where $\gamma=(\beta, \alpha, \sigma), \beta \in V_{m-n}$, $\alpha \in V_{n}$ and $\sigma \in V_{s}$. By the definition of the sequence of a function, $\ell_{1}, \ell_{2}$ and $\ell_{3}$ are the sequences of $\langle\beta, y\rangle,\langle\alpha, x\rangle$ and $\langle\sigma, z\rangle$ respectively.

Suppose that $W(\gamma) \leq k+p+1$. Since $W(\gamma)=W(\beta)+W(\alpha)+W(\sigma)$, we have $W(\alpha)+W(\sigma) \leq k+p+1$, which implies that either $W(\alpha) \leq k$ or $W(\sigma) \leq p$. Recall that $\varphi_{j} \in \Omega_{k, n}$. If $W(\alpha) \leq k, \zeta_{j}$ and $\ell_{2}$ must be orthogonal, and hence $\left\langle\zeta_{j}, \ell_{2}\right\rangle=0$. Otherwise if $W(\sigma) \leq p,\left\langle\xi_{j}, \ell_{3}\right\rangle=0$, since each $f_{j}$ is a $p$ th-order correlation immune function. Thus $\langle\eta, L\rangle=0$. By Lemma $2, g(y, x, z)$ is a $(k+p+1) t h$-order correlation immune function on $V_{m+s}$.

To obtain the nonlinearity of the function $g$, we assume that in the above discussion $h$ is an arbitrary affine function on $V_{m+s}$. Then $L$, the sequence of $h$, can be expressed as $L= \pm \ell_{1} \otimes \ell_{2} \otimes \ell_{3}$, and hence

$$
\langle\eta, L\rangle= \pm\left(c_{0}\left\langle\zeta_{0}, \ell_{2}\right)\left\langle\xi_{0}, \ell_{3}\right\rangle+\cdots+c_{2^{m-n}-1}\left\langle\zeta_{2^{m-n}-1}, \ell_{2}\right\rangle\left\langle\xi_{2^{m-n}-1}, \ell_{3}\right\rangle\right) .
$$

By Lemma 5

$$
\left\langle\xi_{j}, \ell_{3}\right\rangle \leq 2^{s}-2 N_{j j} \leq 2^{s}-2 N .
$$

On the other hand, since the rows of an Hadamard matrix are mutually orthogonal, we have the following result:

$$
\left\langle\zeta_{j}, \ell_{2}\right\rangle= \begin{cases}2^{n} \text { if } \zeta_{j}=\ell_{2} \\ 0 & \text { otherwise }\end{cases}
$$


When there is a $j$ such that $\zeta_{j}=\ell_{2}$, we have $|\langle\eta, L\rangle| \leq t \cdot 2^{n}\left(2^{s}-2 N\right)$. Otherwise if there is no $j$ such that $\zeta_{j}=\ell_{2},|\langle\eta, L\rangle|=0$. In summary, we have $|\langle\eta, L\rangle| \leq$ $t \cdot 2^{n}\left(2^{s}-2 N\right)$. By Lemma $5, d(g, h) \geq 2^{m-1}-t \cdot 2^{n}\left(2^{s-1}-N\right)$. Since $h$ is arbitrary, $N_{g} \geq 2^{m-1}-t \cdot 2^{n}\left(2^{s-1}-N\right)$.

By a similar discussion as in the last part of the proof of Theorem 14, the theorem is true for the more general case when $r(y)=r_{1}(y) \oplus r_{2}(y) \neq 0$.

The construction (12) can be considered as an extension of the construction (5), in the sense that if $s=0$ and each function $f_{j}$ is defined as a constant, the former is reduced to the latter.

\subsection{Direct Sum of Two Correlation Immune Functions}

Lemma 17. Let $f_{1}$ be a $k_{1}$ th-order correlation immune function on $V_{n_{1}}, f_{2}$ be a $k_{2}$ th-order correlation immune function on $V_{n_{2}}$. Then $g(x, y)=f_{1}(x) \oplus f_{2}(y)$ is a $\left(k_{1}+k_{2}+1\right)$ th-order correlation immune function on $V_{n_{1}+n_{2}}$, where' $x=$ $\left(x_{1}, x_{2}, \ldots, x_{n_{1}}\right)$ and $y=\left(y_{1}, y_{2}, \ldots, y_{n_{2}}\right)$.

Proof. Let $\xi_{1}$ and $\xi_{2}$ be the sequences of $f_{1}$ and $f_{2}$ respectively. Then by Lemma 6 , $\eta=\xi_{1} \otimes \xi_{2}$ is the sequence of $g$.

Let $\varphi$ be a linear function on $V_{n_{1}+n_{2}}$. Then $\varphi$ can be written as $\varphi=\langle\gamma, z\rangle=$ $\langle\alpha, x\rangle \oplus\langle\beta, y\rangle$, where $z=(x, y), \gamma=(\alpha, \beta) \in V_{n_{1}+n_{2}}, \alpha \in V_{n_{1}}$ and $\beta \in V_{n_{2}}$. Now let $L$ be the sequence of $\varphi$. By Lemma $3, L$ is a row of $H_{n_{1}+n_{2}}$. Since $H_{n_{1}+n_{2}}=H_{n_{1}} \otimes H_{n_{2}}, L$ can be expressed as $L=\ell_{1} \otimes \ell_{2}$, where $\ell_{1}$ is a row of $H_{n_{1}}$ and $\ell_{2}$ is a row of $H_{n_{2}}$.

Now we show that $\ell_{1}$ matches the sequence of $\langle\alpha, x\rangle$, and $\ell_{2}$ matches the sequence of $\langle\beta, y\rangle$. Assume that $\ell_{1}^{\prime}$ is the sequence of $\langle\alpha, x\rangle$, and $\ell_{2}^{\prime}$ is the sequence of $\langle\beta, y\rangle$. By Lemma 6, $\ell_{1}^{\prime} \otimes \ell_{2}^{\prime}$ is the sequence of $\varphi$. Thus $L=\ell_{1} \otimes \ell_{2}=\ell_{1}^{\prime} \otimes \ell_{2}^{\prime}$. By Lemma $3, \ell_{1}^{\prime}$ is a row of $H_{n_{1}}$ and $\ell_{2}^{\prime}$ is a row of $H_{n_{2}}$. This means that $\ell_{1}=\ell_{1}^{\prime}$ and $\ell_{2}=\ell_{2}^{\prime}$. Put it in another way, $\ell_{1}$ is the sequence of $\langle\alpha, x\rangle$, and $\ell_{2}$ is the sequence of $\langle\beta, y\rangle$.

Now consider $\gamma$ with $W(\gamma) \leq k_{1}+k_{2}+1$. In this case we have either $W(\alpha) \leq$ $k_{1}$ or $W(\beta) \leq k_{2}$. Thus

$$
\langle\eta, L\rangle=\left\langle\xi_{1} \otimes \xi_{2}, \ell_{1} \otimes \ell_{2}\right\rangle=\left\langle\xi_{1}, \ell_{1}\right\rangle\left\langle\xi_{2}, \ell_{2}\right\rangle=0 .
$$

By Lemma 2, $g$ is indeed a $\left(k_{1}+k_{2}+1\right)$ th-order correlation immune function on $V_{n_{1}+n_{2}}$.

Lemma 18. Let $f_{1}$ be a function on $V_{n_{1}}$ and $f_{2}$ be a function on $V_{n_{2}}$. Suppose that their nonlinearities are $N_{f_{1}}=d_{1}$ and $N_{f_{2}}=d_{2}$ respectively. Then the nonlinearity of $g(x, y)=f_{1}(x) \oplus f_{2}(y)$ satisfies $N_{g} \geq d_{1} 2^{n_{2}}+d_{2} 2^{n_{1}}-2 d_{1} d_{2}$.

Proof. Let $\xi_{1}, \xi_{2}, \eta, L, \ell_{1}, \ell_{2}, \varphi$ be the same as in the proof of Lemma 17. Let $\varphi_{1}=\langle\alpha, x\rangle$ and $\varphi_{2}=\langle\beta, y\rangle$.

By Lemma 13, we have

$$
d_{1}=N_{f_{1}} \leq d\left(f_{1}, \varphi_{1}\right)=2^{n_{1}-1}-\frac{1}{2}\left\langle\xi_{1}, \ell_{1}\right\rangle .
$$


Thus

$$
\left\langle\xi_{1}, \ell_{1}\right\rangle \leq 2^{n_{1}}-2 d_{1}
$$

Similarly

$$
\left\langle\xi_{2}, \ell_{2}\right\rangle \leq 2^{n_{2}}-2 d_{2} .
$$

Note that the right sides of (13) and (14) are both positive. Thus

$$
\langle\eta, L\rangle=\left\langle\xi_{1} \otimes \xi_{2}, \ell_{1} \otimes \ell_{2}\right\rangle=\left\langle\xi_{1}, \ell_{1}\right\rangle\left\langle\xi_{2}, \ell_{2}\right\rangle \leq\left(2^{n_{1}}-2 d_{1}\right)\left(2^{n_{2}}-2 d_{2}\right) .
$$

Again by Lemma 13,

$$
d(g, \varphi)=2^{n_{1}+n_{2}-1}-\frac{1}{2}\langle\eta, L\rangle \geq d_{1} 2^{n_{2}}+d_{2} 2^{n_{1}}-2 d_{1} d_{2}
$$

It is easy to see that the right side of (15) is also positive. Thus if $L$ is an affine sequence (i.e. $\varphi$ is an affine function) (15) still holds. Since $\varphi$ is an arbitrary affine function we have

$$
N_{g} \geq d_{1} 2^{n_{3}}+d_{2} 2^{n_{1}}-2 d_{1} d_{2}
$$

Therefore the lemma is true.

Combining Lemmas 17 and 18 and using Lemma 20 of [7] we have

Theorem 19. Let $f_{1}$ be a $k_{1}$ th-order correlation immune function on $V_{n_{1}}$ and $f_{2}$ be a $k_{2}$ th-order correlation immune function on $V_{n_{2}}$. Also suppose that $N_{f_{1}}=d_{1}$ and $N_{f_{2}}=d_{2}$. Then $g(x, y)=f_{1}(x) \oplus f_{2}(y)$ is a $\left(k_{1}+k_{2}+1\right)$ th-order correlation immune function on $V_{n_{1}+n_{3}}$ whose nonlinearity satisfies

$$
N_{g} \geq d_{1} 2^{n_{2}}+d_{2} 2^{n_{2}}-2 d_{1} d_{2}
$$

where $x=\left(x_{1}, x_{2}, \ldots, x_{n_{1}}\right)$ and $y=\left(y_{1}, y_{2}, \ldots, y_{n_{2}}\right)$. In particular $g$ is balanced if either $f_{1}$ or $f_{2}$ is balanced.

\subsection{Combination of Four Correlation Immune Functions}

This section show that from four correlation immune functions, we can obtain a new functions that achieves a higher order of correlation immunity.

Theorem 20. Let $f_{1}$ and $f_{2}$ be pth-order correlation immune functions on $V_{m}$, and let $h_{1}$ and $h_{2}$ be qth-order correlation immune functions on $V_{n}$. Let $\xi_{1}, \xi_{2}$, $\eta_{1}$ and $\eta_{2}$ be the sequences of $f_{1}, f_{2}, h_{1}$ and $h_{2}$ respectively. Let $\zeta$ be a $(1,-1)$ sequence obtained from $\xi_{1}, \xi_{2}, \eta_{1}$ and $\eta_{2}$ in the following way:

$$
\zeta=\frac{1}{2}\left(\xi_{1}+\xi_{2}\right) \otimes \eta_{1}+\frac{1}{2}\left(\xi_{1}-\xi_{2}\right) \otimes \eta_{2}
$$

where + denotes the component-wise integer addition and $\otimes$ denotes the Kronecker product. Then the function corresponding to $\zeta$ is $a(p+q+1)$ th-order correlation immune function on $V_{m+n}$. 
Proof. Similarly to the proof of Lemma 17, we let $\varphi$ be a linear function on $V_{m+n}$ and $L$ be the sequence of $\varphi$. By Lemma 3,L is a row of $H_{m+n}$. In addition, $\varphi$ can be written as $\varphi=\langle\gamma, z\rangle=\langle\alpha, x\rangle \oplus\langle\beta, y\rangle$, where $\gamma=(\alpha, \beta) \in V_{m+n}, \alpha \in V_{m}, \beta \in$ $V_{n}, z=\left(x_{1}, \ldots, x_{m}, y_{1} \ldots, y_{n}\right), x=\left(x_{1}, x_{2}, \ldots, x_{m}\right)$ and $y=\left(y_{1}, y_{2}, \ldots, y_{n}\right)$. Since $H_{m+n}=H_{m} \otimes H_{n} L$ can be expressed as $L=\ell_{1} \otimes \ell_{2}$, where $\ell_{1}$ is a row of $H_{m}$, and $\ell_{2}$ is a row of $H_{n}$. By the same reasoning as in the proof of Lemma 17, it can be shown that $\ell_{1}$ is the sequence of $\langle\alpha, x\rangle$, and $\ell_{2}$ is the sequence of $\langle\beta, y\rangle$. Thus we have

$$
\begin{aligned}
\langle\zeta, L\rangle & =\frac{1}{2}\left\langle\left(\xi_{1}+\xi_{2}\right) \otimes \eta_{1}, \ell_{1} \otimes \ell_{2}\right\rangle+\frac{1}{2}\left\langle\left(\xi_{1}-\xi_{2}\right) \otimes \eta_{2}, \ell_{1} \otimes \ell_{2}\right\rangle \\
& =\frac{1}{2}\left\langle\left(\xi_{1}+\xi_{2}\right), \ell_{1}\right\rangle\left\langle\eta_{1}, \ell_{2}\right\rangle+\frac{1}{2}\left\langle\left(\xi_{1}-\xi_{2}\right), \ell_{1}\right\rangle\left\langle\eta_{2}, \ell_{2}\right\rangle .
\end{aligned}
$$

For $\gamma \in V_{m+n}$ with $W(\gamma) \leq p+q+1$, we have either $W(\alpha) \leq p$ or $W(\beta) \leq q$. This implies that either of the following two situations occurs: $(1)\left\langle\xi_{1}, \ell_{1}\right\rangle=0$ and $\left\langle\xi_{2}, \ell_{1}\right\rangle=0$, and $(2)\left\langle\eta_{1}, \ell_{2}\right)=0$ and $\left\langle\eta_{2}, \ell_{2}\right\rangle=0$. As a consequence, we have $\langle\zeta, L\rangle=0$.

Note that a similar technique to the construction (16) has been used in obtaining higher order Hadamard matrices from lower order Hadamard matrices [6].

\section{Conclusion}

We have studied correlation immune functions using the theory of Hadamard matrices. In particular, we have presented a new method for directly constructing correlation immune functions. It is shown that the method generates the same set of functions as that by a method of Camion et al. The new method is more convenient for use in practice since it allows one to calculate the nonlinearity of functions obtained and to discuss the algebraic degrees and propagation characteristics of the functions. Three methods for obtaining correlation immune functions on a higher dimensional space from known correlation immune functions on a lower dimensional space are also presented. We believe that these various methods of generating correlation immune functions, by direct construction or by combining known correlation immune functions, will find a wide range of applications in computer security.

\section{References}

1. W. Blaser and P. Heinzmann. New cryptographic device with high security using public key distribution. In Proceedings of IEEE Student Paper Contest 1979-1980, pages 145-153, 1982.

2. P. Camion, C. Carlet, P. Charpin, and N. Sendrier. On correlation-immune functions. In Advances in Cryptology: Crypto'g1 Proceeding, volume 576, Lecture Notes in Computer Science, pages 87-100. Springer-Verlag, Berlin-Heidelberg-New York, 1991. 
3. Xiao Guo-zhen and J. L. Massey. A spectral characterization of correlation-imm une combining functions. IEEE Transactions on Information Theory, 34 No. 3:569-571, 1988.

4. R. A. Rueppel. Analysis and Design of Stream Ciphers. Springer-Verlag, Berlin, Heidelberg, New York, London, Paris, Tokyo, Berlin, Heidelberg, New York, London, Paris, Tokyo, 1986. In Communications and Control Engineering Series, Editors: A. Fettweis, J. L. Massey and M. Thoma.

5. R. A. Rueppel. Stream ciphers. In G. J. Simmons, editor, Contemporary Cryptography: the Science of Information Integrity, chapter 2, pages 65-134. IEEE Press, New York, 1992.

6. J. Seberry and M. Yamada. Hadamard matrices, sequences, and block designs. In J. H. Dinitz and D. R. Stinson, editors, Contemporary Design Theory: A Collection of Surveys, chapter 11, pages 431-559. John Wiley \& Sons, Inc, 1992.

7. J. Seberry and X. M. Zhang. Highly nonlinear 0-1 balanced functions satisfying strict avalanche criterion. Presented at AUSCRYPT'92, 1992.

8. T. Siegenthaler. Correlation-immunity of nonlinear combining functions for cryptographic applications. IEEE Transactions on Information Theory, IT-30 No. 5:776$779,1984$.

9. W. D. Wallis, A. Penfold Street, and J. Seberry Wallis. Combinatorics: Room Squares, sum-free sets, Hadamard Matrices, volume 292 of Lecture Notes in Mathematics. Springer-Verlag, Berlin-Heidelberg-New York, 1972. 